\title{
New information on sexual dimorphism and allometric growth in Keichousaurus hui, a pachypleurosaur from the Middle Triassic of Guizhou, South China
}

Yifan Xue, Dayong Jiang, Ryosuke Motani, Olivier Rieppel, Yuanlin Sun, Zuoyu Sun, Cheng Ji, and Pengfei Yang

Acta Palaeontologica Polonica 60 (3), 2015: 681-687 doi:http://dx.doi.org/10.4202/app.00006.2013

Keichousaurus hui is a small pachypleurosaur (Reptilia: Sauropterygia) from the Triassic of China. Many specimens of various growth stages are known, making them ideal for ontogenetic research. We report 22 new specimens from the Middle Triassic of Xingyi (Guizhou, south China), and combined their skeletal measurements with those from 85 published specimens to analyze the ontogenetic trajectory of sexual dimorphism. An Exploratory Factor Analysis suggests that the largest factors behind morphological disparity within the species are body size followed by gender. Sexual dimorphism is most clearly reflected in selected skeletal ratios that are more pronounced in males than in females. We found that the relative length of femur to body size was useful in gender identification, in addition to three ratios that are traditionally used, namely a distal expansion of the humerus relative to its shaft, humerus length relative to body size, and humerus length relative to femur length. Two distinctive patterns exist in allometric changes of these four ratios. The distal expansion of the humerus is exceptional in that it is equally pronounced in juvenile and adult males and therefore must have been fully established during embryonic growth. The other three features are not pronounced at birth size and subsequently become pronounced during postembryonic growth. However, males and females already show different growth trajectories at birth size even in these three. Therefore, the fate of sexually dimorphic features seems to have already been set during embryonic growth in $K$. hui.

Key words: Pachypleurosauria, Sauropterygia, Keichousaurus hui, allometry, sexual dimorphism, Triassic, China.

Xue Yifan [xueyifan1992@sina.com], Sun Yuanlin [ylsun@pku.edu.cn], Sun Zuoyu [sunzuoyu@pku.edu.cn], and Yang Pengfei [445872056@qq.com], Key Laboratory of Orogenic Belt and Crustal Evolution, MOE; Department of Geology and Geological Museum, Peking University, Beijing 100871, PR China; Jiang Dayong [djiang@pku.edu.cn] (corresponding author) and Ji Cheng [jicheng04@126.com ], Key Laboratory of Orogenic Belt and Crustal Evolution, MOE; Department of Geology and Geological Museum, Peking University, Beijing 100871, PR China; State Key Laboratory of Palaeobiology and Stratigraphy (Nanjing Institute of 
Geology and Palaeontology, CAS), Nanjing 210008, PR China; Ryosuke Motani [rmotani@ucdavis.edu ] (corresponding author), Department of Earth and Planetary Sciences,

University of California, Davis, California 95616, USA; Olivier Rieppel [orieppel@ fieldmuseum.org], Center of Integrative Research, Field Museum of Natural History, Roosevelt Road at Lake Shore Drive, Chicago, Illinois 60615, USA.

This is an open-access article distributed under the terms of the Creative Commons Attribution License (for details please see creativecommons.org), which permits unrestricted use, distribution, and reproduction in any medium, provided the original author and source are credited.

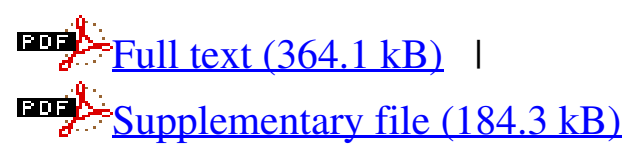

